\title{
Sistematização da origem, da distribuição e dos territórios da artéria cerebral caudal na superfície do encéfalo em gatos
}

\author{
Systematization of the origin, distribution and territory of the caudal cerebral artery on the \\ surface of the encephalon in cats
}

\author{
Eduardo Maurício Mendes de Lima ${ }^{\mathrm{I}}$ Irvênia Luiza de Santis Prada ${ }^{\mathrm{II}}$ \\ Frederico Ozanam Carneiro e Silva ${ }^{\text {III }}$ Renato Souto Severino ${ }^{\text {III }}$ André Luis Quagliatto Santos ${ }^{\text {III }}$ \\ Bárbara Oliveira Borges ${ }^{\mathrm{I}}$ Tiago do Prado Paim ${ }^{\mathrm{I}}$ André Rodrigues da Cunha Barreto Vianna
}

RESUMO

\begin{abstract}
Foram estudados 26 encéfalos de gatos, adultos, sem diferenciação de sexo, sem raça definida, corados com látex e fixados por solução aquosa de formaldeído. As artérias cerebrais caudais, direita e esquerda, apresentaram-se únicas em 96,1 e $88,4 \%$ dos casos, respectivamente. Em $69,2 \%$ dos casos no antímero direito e $80,8 \%$ no esquerdo, a artéria cerebral caudal originou-se pela anastomose entre o ramo caudal da artéria carótida interna, com maior contribuição, $e$ o ramo terminal da artéria basilar. Em $88,4 \%$ dos casos no antímero direito e em $84,6 \%$ no esquerdo, a artéria cerebral caudal originou a artéria tectal rostral e um ramo caudal. $O$ ramo caudal bifurcou-se e vascularizou os colículos rostrais e caudais dos corpos quadrigêmeos e em alguns casos contribuiu na formação do plexo coroide do terceiro ventrículo. A artéria tectal rostral seguiu ventralmente ao lobo piriforme e durante seu trajeto liberou ramos para o hipocampo e, no antímero esquerdo, supriu a superfície dorsal do tálamo, contribuindo para a formação do plexo coroide do terceiro ventrículo.
\end{abstract}

Palavras-chave: encéfalo, artéria cerebral caudal, Felis catus domesticus.

\section{ABSTRACT}

In this study were used 26 brains of adult cats, without sex differentiation and definable race, colored with latex and fixed in formaldehyde aqueous solution. The right and left caudal cerebral arteries were single in $96.1 \%$ and $88.4 \%$ of the sample, respectively. In $69.2 \%$ of the samples on the right side and $80.8 \%$ on the left, the caudal cerebral artery fin from the anastomosis behind the caudal branch of the internal carotid artery, with a large contribution, and the basal artery terminal branch. In $88.4 \%$ of the samples on the right side and $84.6 \%$ on the left, the caudal cerebral artery showed the tectal rostral artery and one caudal branche. The caudal branch forked in two branches that supplied the rostral and caudal colliculis of the quadruplet bodies and formed the choroid plexus of the third ventricle. The tectal rostral artery followed ventrally to the piriform lobe and during his way sent some branches to hippocampus. In the left side, this vessel supplied the dorsal surface of thalamus and contributed to the formation of the choroid plexus of the third ventricle.

Key words: encephalon, caudal cerebral artery, Felis catus domesticus.

\section{INTRODUÇÃO}

Durante o contínuo processo de evolução do sistema nervoso central, foi possível observar a existência de constantes modificações na configuração dos vasos responsáveis por sua vascularização (LIMA et al., 2006). O estudo morfofuncional do sistema nervoso central vem demonstrando aspectos interessantes e pouco explorados, em especial aqueles relativos ao suprimento sanguíneo para o encéfalo. Nesse contexto, verificou-se que ramos arteriais não supriram somente aquelas estruturas que originaram as suas denominações, mas também participaram significantemente do suprimento sanguíneo de outras estruturas importantes (CUNHA et al., 2001).

'Faculdade de Agronomia e Medicina Veterinária, Instituto Central de Ciências (ICC), Universidade de Brasília (UnB), Ala Sul, Campus Universitário Darcy Ribeiro, CP 4508, 70760-701, Brasília, DF, Brasil. E-mail: limaemm@unb.br. *Autor para correspondência.

"Faculdade de Medicina Veterinária e Zootecnia (FMVZ), Universidade de São Paulo (USP), São Paulo, SP, Brasil.

II'Faculdade de Medicina Veterinária, Universidade Federal de Uberlândia (UFU), Belo Horizonte, MG, Brasil. 
É notório que vários autores abordaram a vascularização do encéfalo das mais diversas espécies animais, tais como ALCÂNTARA et al. (2000) e HEINZEN (2000) em cães, LINDEMANN \& CAMPOS (2004) em gambás, CAMPOS (2007) em chinchilas e DEPEDRINI \& CAMPOS (2007) em graxaim-do-campo, porém poucos autores relataram o suprimento arterial do encéfalo em gatos (LIMA et al., 2006).

Diante disso, verificou-se que ainda há muito a ser explorado acerca do sistema vascular cerebral dos gatos pela sua singularidade, mais especificamente em virtude da complexidade de arranjos e disposição das artérias encefálicas em relação à maioria das outras espécies de canídeos, felídeos e roedores NANDA (1986), ALCÂNTARA \& PRADA (1996), ALCANTÂRA et al. (2000), DEPEDRINI \& CAMPOS (2003), RECKZIEGEL et al. (2004), LIMA et al. (2006), CAMPOS (2007) e DEPEDRINI \& CAMPOS (2007) e principalmente no que tange ao grande número de fontes de suprimento sanguíneo verificado nessa espécie. Dessa forma, objetivou-se conhecer a origem, a distribuição e os territórios da artéria cerebral caudal na superfície do encéfalo desses animais.

\section{MATERIAL E MÉTODOS}

Foram estudados 26 encéfalos de gatos (Felis catus domesticus), sem raça definida, adultos, de ambos os sexos, obtidos após óbito natural junto ao Centro de Controle de Zoonoses de Brasília e de Clínicas Veterinárias. O sistema arterial da cabeça, por meio da canulação da artéria carótida comum direita, foi preenchido com solução aquosa, a $50 \%$, de Neoprene Látex “450" corada com pigmento específico. Em seguida, foram fixados o encéfalo e a porção cervical da medula espinhal a partir da imersão destes em recipientes contendo solução aquosa de formol, a 10\%, por um período mínimo de 48 horas.

Após a fixação, os encéfalos foram retirados da cavidade craniana e a porção cervical da medula espinhal foi retirada do canal vertebral. A partir dessa etapa, foram realizadas as dissecações e observados os diferentes arranjos com o auxílio de lupa.

A nomenclatura adotada esteve, de modo geral, de acordo com o preconizado pelo INTERNATIONAL COMMITTEE ON VETERINARY GROSS ANATOMICALNOMENCLATURE (2005).

\section{RESULTADOS E DISCUSSÃO}

\author{
De acordo com DEPEDRINI \& CAMPOS \\ (2003), CAMPOS (2007) e DEPEDRINI \& CAMPOS \\ (2007) em chinchilas e graxaim-do-campo, os animais
}

deste estudo tiveram o encéfalo suprido pelos sistemas carótico e vértebro-basilar. Nos gatos investigados, a artéria cerebral caudal foi considerada como um colateral do ramo caudal da artéria carótida interna (Figura 1), bem como verificado por LIMA et al. (2006), mas com uma denominação diferenciada, uma vez que esses autores consideraram tal vaso como sendo um ramo colateral da artéria carótida do encéfalo.

No mesmo contexto, dois ramos terminais oriundos da artéria carótida internos bifurcaram-se profundamente em ramos rostral e caudal de acordo com TANDLER (1898), GILLILAN (1976) e ALCANTARA\& PRADA (1996). Em parte, isso foi ao encontro com o observado nos animais investigados, pois, apesar de contextos de nomenclatura diferentes, a fonte supridora e originária da artéria cerebral caudal foi o sistema carótico, acrescido do sistema vértebrobasilar.

Origens distintas poderiam ser apresentadas pela artéria cerebral caudal, ocorrendo como um ramo terminal da artéria basilar, como verificado por RECKZIEGEL et al. (2004) em capivaras, por meio de um vaso único, duplo ou triplo. Tais arranjos não foram visualizados em gatos, sobretudo a artéria cerebral caudal nesses animais originaram-se do ramo caudal da artéria carótida interna, que, de acordo com os achados do presente estudo e de NANDA (1986), ALCÂNTARA et al. (2000), LIMA et al. (2006), CAMPOS (2007) e DEPEDRINI \& CAMPOS (2007), tiveram origem de um complexo de anastomoses entre os sistemas carótico e vértebro-basilar. De outra forma, em $69,2 \%$ dos casos no antímero direito e $80,8 \%$ no esquerdo, a artéria cerebral caudal originou-se da anastomose entre o ramo caudal da artéria carótida

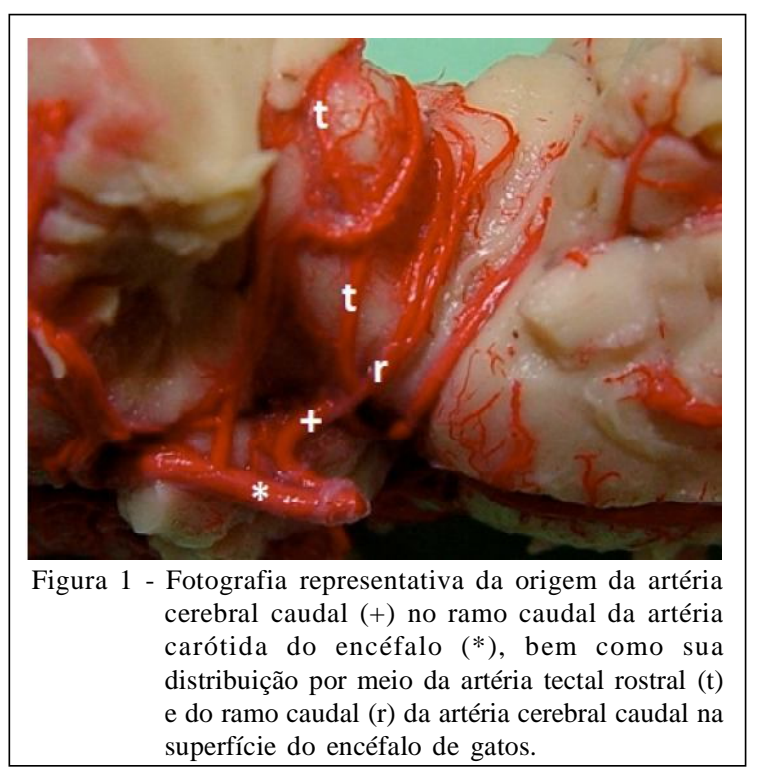


interna, com maior contribuição, e o ramo terminal da artéria basilar. As variações ocorreram por aumento no diâmetro do ramo terminal da artéria basilar, em $23,1 \%$ dos casos no antímero direito e $3,8 \%$ no esquerdo, ou quando uma dessas duas artérias originava-se isoladamente de um dos ramos da artéria cerebral caudal, como verificado em $7,7 \%$ dos casos no antímero direito e $15,4 \%$ no esquerdo.

As artérias cerebrais caudais, direita e esquerda, apresentaram-se únicas em 96,1 e 88,4\% dos casos, respectivamente (Figura 1); e sempre localizadas na face ventral do pedúnculo cerebral, rostralmente ao nervo oculomotor. Esse achado está de acordo com o que foi descrito por JENKINS (1978) e HEINZEN (2000) para cães. De outra forma, WILAND (1973) citou que, em 68 cães, imediatamente antes de penetrar no lobo piriforme, a artéria cerebral caudal se dividiu em dois ramos; no entanto, esses autores não nominaram especificamente essas ramificações. Já DEPEDRINI \& CAMPOS (2007), em graxaim-do-campo, consideraram a presença da artéria tectal rostral. Esse resultado foi ao encontro dos achados deste estudo para os gatos domésticos, pois nesses animais foi possível verificar que a artéria cerebral caudal, em cada um dos antímeros, originou a artéria tectal rostral. Esse vaso foi tido como o principal ramo da artéria cerebral caudal.

Nos gatos, a artéria cerebral caudal, em ambos os antímeros, cedeu ramos corticais e terminais ao longo do encéfalo, através da emissão da artéria tectal rostral e de um ramo caudal (Figura 1). Em relação ao trajeto, em $88,4 \%$ dos casos no antímero direito e $84,6 \%$ no antímero esquerdo, a artéria cerebral caudal dividiu-se em dois ramos, sendo que a artéria tectal rostral seguiu ventralmente ao lobo piriforme, já o ramo caudal dividiu-se em dois outros ramos. Destes, o ramo caudal supriu, principalmente, o colículo rostral, mas também emitiu ramos para o colículo caudal. A artéria tectal rostral liberou ainda ramos que contribuíram para formação do plexo coroide do terceiro ventrículo. Esses ramos supriram a superfície dorsal do tálamo em 7,7\% dos casos no antímero direito e $11,5 \%$ no esquerdo, para a superfície do hemisfério cerebral em 3,8\% dos casos no antímero direito e o corpo geniculado medial em 3,8\% dos casos à esquerda. $\mathrm{O}$ trajeto desses ramos não exibiu um padrão, alcançando o pedúnculo cerebelar médio, a região dorsal do tálamo, a superfície do hemisfério cerebral, o corpo mamilar e o corpo geniculado medial.

Em se tratando dos ramos corticais e terminais da artéria cerebral caudal, nos gatos estudados, esses ramos distribuíram-se da mesma forma que em cães, equinos e ruminantes, como descrito por NANDA (1986), e também em capivaras, como apresentado por RECKZIEGEL et al. (2004). Nesse contexto, destaca-se que os achados nos gatos foram ao encontro dos relatos de ALCÂNTARA et al. (2000) e RECKZIEGEL et al. (2004) pelo fato de que a artéria cerebral caudal após sua origem seguiu distribuindose ao longo do teto do mesencéfalo.

Como ALCÂNTARA et al. (2000) para cães, foi observada, em todos os gatos, a presença de um ramo colateral distribuindo-se nos colículos rostrais e caudais dos corpos quadrigêmeos. Já DEPEDRINI \& CAMPOS (2007), em graxaim-do-campo, consideraram que o primeiro ramo colateral emitido pela artéria cerebral caudal foi a artéria tectal rostral, vaso que se distribuiu no colículo rostral e na porção mais rostral do colículo caudal.

Foi possível verificar que, nos gatos, a artéria cerebral caudal percorreu toda a borda do hipocampo, cedendo ramos para este, fato que foi de encontro com os relatos realizados por NILGES (1944) em guinea pigs, cães, coelhos, gatos e macacos.

Nos animais deste estudo, o trajeto da artéria cerebral caudal foi o mesmo que em gambás, como citado por LINDEMANN \& CAMPOS (2004). Esse vaso avançou pela face dorsal do tálamo em sentido rostromedial, emitindo, durante esse percurso, uma série de pequenos ramos mediais que se distribuíram para o dorso do tálamo, o corpo pineal e a tela corioide do terceiro ventrículo.

A artéria tectal rostral seguiu ventralmente o lobo piriforme e dividiu-se em dois ramos em $84,6 \%$ dos casos no antímero direito e 92,3\% no antímero esquerdo. Em 66,7\% dos casos, no antímero esquerdo, esses ramos contornaram o corpo geniculado lateral e, em seguida, cruzaram-se, invertendo suas posições originais. Já no antímero direito, observou-se que, em $68,2 \%$ dos casos, os ramos originários da artéria tectal rostral correspondente contornavam o corpo geniculado lateral, mas não o cruzavam. No entanto, ao longo de seu trajeto, emitiram pequenos ramos para o hipocampo.

Em outro contexto e de acordo com LIMA et al. (2006), a artéria mesencefálica originou-se a partir do tronco comum com a artéria cerebral caudal. Entretanto, nos gatos deste estudo, esse vaso não emitiu ramos somente para os colículos rostral e caudal dos corpos quadrigêmeos, mas também contribuiu para a formação do plexo coroide do terceiro ventrículo. Além disso, em mais de $80 \%$ dos casos, essas artérias originaram-se a partir de um tronco comum. Por esses motivos, foi possível entender que essa artéria foi, na realidade, um ramo caudal da artéria cerebral caudal, diferentemente do relatado por LIMAet al. (2006). Ainda nesse contexto, no que se refere à nomenclatura 
adotada, podem ser citadas as descrições realizadas em cães por NANDA (1986) e HEINZEN (2000), quando denominaram esse mesmo ramo como sendo o ramo coroide caudal, diferentemente do observado neste estudo.

O território de vascularização da artéria cerebral caudal nos gatos mostrou-se constante, coincidindo assim com os informes de NILGES (1944), NANDA (1986), ALCÂNTARA et al. (2000), RECKZIEGEL et al. (2004), CAMPOS (2007) e DEPEDRINI \& CAMPOS (2007). Destaca-se que, em $53,8 \%$ dos casos no antímero esquerdo, os ramos supriram a superfície dorsal do tálamo e contribuíram para a formação do plexo coroide do terceiro ventrículo. Variações foram verificadas por ramos que alcançaram apenas o plexo coroide do terceiro ventrículo em 19,2\% dos casos, a superfície dorsal do tálamo em 3,8\%, o plexo coroide do terceiro ventrículo e o colículo rostral em 3,8\% e o colículo rostral e a superfície dorsal do tálamo em 19,2\%.

A artéria cerebral caudal cedeu um variável número de ramos corticais que se distribuíram caudalmente. Esses ramos corticais estiveram distribuídos na face caudal do lobo piriforme e na superfície tentorial dos hemisférios cerebrais, bem como na superfície dorsolateral ao nível do sulco marginal. Os territórios da artéria cerebral caudal incluíram o tálamo, o colículo rostral, parte do colículo caudal, a face caudal do lobo piriforme, a superfície tentorial, a porção retroesplênica e a face medial ao redor da área da superfície dorsolateral dos hemisférios cerebrais, ao longo das margens das fissuras longitudinal e transversal do cérebro.

Esses dados estão de acordo com NANDA (1986) em cães, em que a artéria caudal do cérebro emitiu ramos centrais e corticais, para o corpo geniculado lateral, o tálamo, o corpo pineal, o plexo coroide do terceiro ventrículo, o corpo geniculado medial, o braço do colículo rostral e a área talâmica médiocaudal.

Os achados neste estudo coincidiram ainda com os relatos de LINDEMANN \& CAMPOS (2004) em gambás, quando o território de vascularização da artéria cerebral caudal compreendeu o hipocampo caudal, os dois terços rostrais do tecto mesencefálico, as porções dorsais e laterais do tálamo e a face caudal do lobo piriforme.

Da mesma forma, para DEPEDRINI \& CAMPOS (2007), a artéria cerebral caudal em graxaimdo-campo teve como território de vascularização o pólo caudal, o terço medial e caudal da superfície dos hemisférios cerebrais e o tálamo e, através da artéria tectal rostral, supriu o teto do mesencéfalo, mas não se distribuiu na porção caudal do colículo caudal e cerebelo.

\section{CONCLUSÃO}

As artérias cerebrais caudais originaram-se por meio de um vaso único e em cada antímero estiveram dispostas na face ventral do pedúnculo cerebral e cederam a artéria tectal rostral e um ramo caudal. Em $69,2 \%$ dos casos no antímero direito e $80,8 \%$ no antímero esquerdo, a artéria cerebral caudal foi formada pela anastomose entre o ramo caudal da artéria carótida interna, com maior contribuição, e o ramo terminal da artéria basilar.

A artéria tectal rostral dividiu-se em dois ramos em $84,6 \%$ dos casos no antímero direito e $92,3 \%$ no antímero esquerdo e apresentaram um trajeto sinuoso em $66,7 \%$ dos casos no antímero, cruzando-se no corpo geniculado lateral. No antímero direito, em $68,2 \%$ dos casos, esses ramos apresentaram um trajeto retilíneo. A artéria tectal rostral, de cada um dos antímeros, cedeu ramos para o hipocampo, e a artéria tectal rostral esquerda supriu a superfície dorsal do tálamo e contribuiu ainda para a formação do plexo coroide do terceiro ventrículo. Já no antímero direito não foi possível estabelecer um padrão exato para os territórios vascularizados por essa artéria. Com relação aos ramos caudais da artéria cerebral caudal, foi possível verificar que estes supriram os colículos rostral e caudal dos corpos quadrigêmeos e o plexo coroide do terceiro ventrículo, na maioria dos casos.

\section{COMITÊ DE ÉTICA E BIOSEGURANÇA}

Esta pesquisa foi analisada e aprovada pelo Comitê de ética no uso animal da Universidade de Brasília, sob protocolo número 40917/2007.

\section{FONTE FINANCIADORA}

Este estudo foi financiado pela Fundação de Empreendimentos Científicos e Tecnológicos.

\section{REFERÊNCIAS}

ALCÂNTARA, M.A.; PRADA, I.L.S. Artérias da base do encéfalo em cães (Canis familiaris, Linnaeus 1758). I. estudo anatômico de suas origens e comportamento. Brazilian Journal of Veterinary Research and Animal Science, São Paulo, v.33, p.67-71, 1996

ALCÂNTARA, M.A. et al. A artéria cerebral caudal em cães (Canis familiaris, Linnaeus 1758) SRD - estudo da anatomia de seus segmentos e distribuição. Ciência Cultura, Tuiuti, v.21, p.57-69, 2000.

CAMPOS, R. Systematization, distribution and territory of the caudal cerebral artery on the surface of the brain in chinchilla 
(Chinchilla lanigera). Brazilian Journal of Morphological Sciences, Campinas, v.24, p.180-186, 2007.

CUNHA, I.P. et al. Comportamento anatômico da artéria cerebelar média no cão (Canis familiaris, Linnaeus, 1758). Veterinária Notícias, Uberlândia, v.7, n.2, p.13-22, 2001.

DEPEDRINI, J.S.; CAMPOS, R. A systematic study of the brain base arteries in the pampas fox (Dusicyon gymnocercus). Brazilian Journal of Morphological Sciences, Campinas, v.20, n.3, p.181-188, 2003.

DEPEDRINI, J.S.; CAMPOS, R. Systematization, distribution and territory of the caudal cerebral artery on the surface of the brain in pampas foxes (Pseudalopex gymnocercus). Brazilian Journal of Morphological Sciences, Campinas, v.24, n.2, p.126-136, 2007.

GILLILAN L.A. Extra- and intra-cranial blood sopply to brains of $\operatorname{dog}$ and cat. American Journal of Anatomy, Salt Lake City, v.146, p.237-254, 1976. Disponível em: <http:// onlinelibrary.wiley.com/doi/10.1002/aja.1001460303/ abstract;jsessionid=7DA0D5F809A0CC6963B4FC23C37069DB.d03t01>. Acesso em: 18 ago. 2010. doi: 10.1002/aja.1001460303.

HEINZEN, R.P.S. Comportamento anatômico da artéria cerebral caudal no cão sem raça definida (Canis familiaris, Linnaeus, 1758). 2000. 115f. Dissertação (Mestrado em Neurociências) - Curso de Pós-graduação em Neurociências, Universidade Federal de Santa Catarina, SC.

INTERNATIONAL COMMITTEE ON VETERINARY GROSS ANATOMICAL NOMENCLATURE. Nomina anatomica veterinaria. 5.ed. Hannover, 2005. 166p.
JENKINS, T.W. Functional mammalian neuroanatomy. 2.ed. Philadelphia: Lea \& Febiger, 1978. p.69-84.

LIMA, E.M.M. et al. Estudo anatômico das artérias da base do encéfalo em gatos. Ars Veterinária, Jaboticabal, v.22, n.1, p.001-007, 2006.

LINDEMANN, T.; CAMPOS, R. Distribuição e território das artérias cerebelares no encéfalo do gambá (Didelphis albiventris). Ciência Rural, Santa Maria, v.34, n.2, p.407412, 2004. Disponível em: http://www.lume.ufrgs.br/bitstream/ handle/10183/22178/000468967.pdf?sequence=1 >. Acesso em: 18 ago. 2010. doi: 10.1590/S0103-84782004000200011.

NANDA, B.S. Suprimento sangüíneo do cérebro. In: GETTY, R. Sisson/Grossman anatomia dos animais domésticos. 5.ed. Rio de Janeiro: Guanabara Koogan, 1986. V.2, p.15131521 .

NILGES, R.G. The arteries of the mammalian cornuammonis. Journal of Comparative Neurology, New Jersey, v.80, p.177190, 1944.

RECKZIEGEL, S.H. et al. Anatomy of the caudal cerebral artery on the surface of capybara (Hydrochoerus hydrochaeris) brain. Brazilian Journal of Morphological Sciences, Campinas, v.21, n.3, p.131-134, 2004.

TANDLER, J. Zur vergleichenden anatomie der Kopfarterien bein den Mammalia. Denkschriften Akademie der Wissenschaften in Wien, Wien, v.67, p.677-784, 1898.

WILAND, D. Variation of the basal arteries of the brain in dogs. Folia Morphologica, Warszawa, v.32, p.63-70, 1973. 